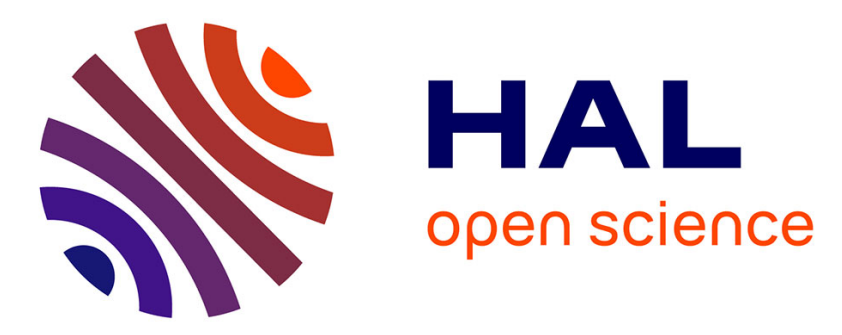

\title{
Caractérisation hydrodynamique d'un sol encroûté en zone sahélienne. 1. Approche expérimentale in situ
}

Pascal Perez, Pierre Todoroff, Jaodat Touma, Michel Fortier

\section{To cite this version:}

Pascal Perez, Pierre Todoroff, Jaodat Touma, Michel Fortier. Caractérisation hydrodynamique d'un sol encroûté en zone sahélienne. 1. Approche expérimentale in situ. Agronomie, 1999, 19 (5), pp.331340. hal-00885934

\author{
HAL Id: hal-00885934 \\ https://hal.science/hal-00885934
}

Submitted on 1 Jan 1999

HAL is a multi-disciplinary open access archive for the deposit and dissemination of scientific research documents, whether they are published or not. The documents may come from teaching and research institutions in France or abroad, or from public or private research centers.
L'archive ouverte pluridisciplinaire HAL, est destinée au dépôt et à la diffusion de documents scientifiques de niveau recherche, publiés ou non, émanant des établissements d'enseignement et de recherche français ou étrangers, des laboratoires publics ou privés. 


\title{
Caractérisation hydrodynamique d'un sol encroûté en zone sahélienne. 1. Approche expérimentale in situ
}

\author{
Pascal Perez ${ }^{\mathrm{a}^{*}}$, Pierre Todoroff ${ }^{\mathrm{a}}$, Jaodat Touma ${ }^{\mathrm{b}}$, Michel Fortier ${ }^{\mathrm{a}}$

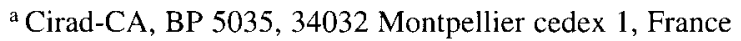 \\ ${ }^{\mathrm{b}}$ Orstom-DEC, BP 5045, 34032 Montpellier cedex 1, France \\ (Reçu : 30 juin 1998, accepté : 16 mars 1999)
}

\begin{abstract}
Determining the hydraulic properties of a Sahelian crusted soil. 1. In field experiment and measurements. Surface sealing affects considerably the infiltration capacity of soils in the Sahelian zone. Due to experimental constraints, in situ determination of the hydraulic resistance of soil crusts is seldom attempted. A methodology is presented that allowed the determination of the hydrodynamic characteristics of a ferrugineous soil located in Senegal and their evolution under two successive simulated rains. Values inferred from tensiometric and capacitive measurements are, to some extent, in agreement with those determined by tension disk infiltrometer at the end of the experiment; however, only the former method permits the assessment of the crust resistance. The structural modification of the soil surface is confirmed by mercury intrusion on undisturbed soil samples. (@ Inra/Elsevier, Paris.)
\end{abstract}

crusted soil / rain simulator / tension disk infiltrometer / mercury intrusion / hydraulic resistance

Résumé - La présence d'une croûte superficielle a une influence prépondérante sur les capacités d'infiltration de la plupart des sols en zone sahélienne. La caractérisation, in situ, de la résistance hydraulique développée au sein de cette croûte a longtemps été limitée par des contraintes métrologiques. Le protocole expérimental présenté nous a permis de suivre l'évolution du comportement hydrodynamique d'un sol ferrugineux du Sénégal soumis à deux pluies artificielles. On observe une certaine cohérence entre les résultats des mesures tensio-capacitives effectuées durant les averses et ceux fournis par l'infiltrométrie à succion contrôlée en fin d'expérimentation. Cependant, seule la première technique permet d'accéder à la conductivité hydraulique propre de la croûte. L'analyse d'échantillons de sol non remaniés par porosimétrie au mercure confirme la modification structurale qui intervient à la surface du sol. (C Inra/Elsevier, Paris.)

sol encroûté / simulateur de pluie / infiltromètre à succion contrôlée / porosimétrie au mercure / résistance hydraulique 


\section{Introduction}

La présence de croûtes superficielles est une caractéristique de la plupart des sols en zone sahélienne $[8,17]$. Il a été démontré que leur capacité d'infiltration était davantage liée à des critères d'états de surface (couvert végétal, activité faunique, organisations pelliculaires) qu'à la nature du profil pédologique [2]. Dans le cas des sols cultivés, les états de surface évoluent principalement en fonction des facteurs climatiques et des pratiques culturales et en retour influencent durablement les conditions d'alimentation hydrique des cultures [ 1 , $20,24]$.

La mise en évidence, in situ, des propriétés hydrodynamiques d'un sol soumis à l'encroûtement a longtemps été limitée par des contraintes métrologiques. Mc Intyre [18], puis Bresler et Kemper [6], travaillant en laboratoire et sous pluies simulées, ont fourni des valeurs de conductivité hydraulique de la croûte 200 à 2000 fois plus faibles que celles des sols en place.

Dans un premier temps, les expérimentations sous pluies simulées, menées en Afrique de l'Ouest, ont permis de développer des lois empiriques d'infiltration ou de ruissellement $[9,26]$. Des solutions techniques récentes permettent d'envisager des protocoles de mesures beaucoup plus précis. Ainsi, l'association, sous pluies simulées, de la tensiométrie et de l'humidimétrie capacitive offre de nouvelles possibilités concernant le suivi des processus d'infiltration $[14,27]$. D'autre part, la caractérisation hydrodynamique des états initiaux et finaux peut-être réalisée grâce à un infiltromètre à succion contrôlée [21, 31]. Enfin, les techniques de porosimétrie au mercure fournissent des informations pertinentes sur l'évolution structurale de l'horizon superficiel [12].

Cette étude présente le dispositif expérimental utilisé pour caractériser, in situ, l'évolution superficielle d'un sol ferrugineux tropical. L'expérimentation a été menée sous pluies simulées. L'état initial et l'état final ont été caractérisés à la fois par infiltrométrie à succion controlée et par prélèvement d'échantillons, soumis ultérieurement à une analyse par porosimétrie au mercure. Le comportement hydrodynamique a été suivi grâce à un dispositif tensio-capacitif. La conductivité de la croute a été évaluée de deux manières : (i) par la formule de Hillel et Gardner, en utilisant les mesures de potentiel sous pluie, (ii) par analyse du régime transitoire sous infiltromètre.

\section{Matériel et méthodes}

\subsection{Le site}

L'expérimentation s'est déroulée durant la saison sèche sur la station de l'Institut Sénégalais de Recherches Agricoles (ISRA) à Kaolack, Sénégal. Le sol appartient au groupe des sols ferrugineux tropicaux faiblement lessivés, développés sur un manteau sableux quaternaire [5]. Il est peu évolué et présente un profil homogène, à structure massive. L'horizon superficiel (0 à $25 \mathrm{~cm}$ de profondeur) est pauvre en matières organiques $(0,5 \%)$. La texture est dominée par des sables fins $(61,6 \%)$ et des limons grossiers $(12,8 \%)$ mais contient peu d'argile (5\%), essentiellement des kaolinites.

La parcelle d'étude correspond à une jachère de un an dont une partie a été défrichée et aplanie un mois avant le début de l'essai. La pente générale est inférieure à $0,3 \%$. À l'état initial, le sol présente une surface lisse recouverte par endroit d'agrégats de taille inférieure à $5 \mathrm{~mm}$. L'humidité volumique résiduelle du sol n'excède pas $0,03 \mathrm{~cm}^{3} \mathrm{~cm}^{-3}$ jusqu'à $50 \mathrm{~cm}$ de profondeur, le profil initial est donc considéré comme uniformément sec. La densité apparente estimée à partir de prélèvements au cylindre $\left(100 \mathrm{~cm}^{3}\right)$ avoisine 1,60 .

La caractérisation de l'état final a été réalisée une semaine après la fin du protocole de pluie simulée. Le sol est alors ressuyé $\left(\Theta=0,08 \mathrm{~cm}^{3} \mathrm{~cm}^{-3}\right)$ et la surface présente une couleur grisâtre entrecoupée de plages sableuses plus claires.

\subsection{Le dispositif}

\subsubsection{Le simulateur de pluie}

Après un relevé micro-topographique, une parcelle de ruissellement $\left(1 \times 1 \mathrm{~m}^{2}\right)$ a été installé dans la zone défrichée. Le simulateur de pluie est constitué d'un système d'arrosage fixé au sommet d'une tour pyramidale de 4 mètres de haut et protégé de l'action du vent par une 
bâche amovible [7]. L'aspersion est assurée par un gicleur calibré monté sur un bras oscillant dont le mouvement est imprimé par un moteur électrique. La gamme d'intensités disponibles varie de 30 à $150 \mathrm{~mm} \cdot \mathrm{h}^{-1}$ [4]. La tour est centrée sur la parcelle de ruissellement. Cette dernière est reliée à une cuve de réception, elle-même surmontée d'un limnigraphe (figure 1). La précision de mesure nominale est de l'ordre de $0,1 \mathrm{~mm}$ pour les hauteurs d'eau et de 10 secondes pour les temps.

Le protocole de simulation comprend deux pluies séparées par une période de ressuyage de $20 \mathrm{~h}$. Chaque pluie comprend deux averses, l'une de $40 \mathrm{~mm} \cdot \mathrm{h}^{-1}$ pendant $1 \mathrm{~h}$, suivie d'un arrêt de $0,5 \mathrm{~h}$, l'autre de $120 \mathrm{~mm} \cdot \mathrm{h}^{-1}$ pendant $0,5 \mathrm{~h}$; soient $100 \mathrm{~mm}$ précipités. Ces intensités et les énergies unitaires obtenues, de l'ordre de $17 \mathrm{~J} . \mathrm{mm}^{-1} \cdot \mathrm{m}^{-2}$, correspondent à celles rencontrées dans la zone d'étude pour un évènement pluvieux de récurrence décennale [20]. Le flux d'infiltration (F) est calculé à partir des flux précipités $(P)$ et ruisselés $(R)$ déterminés à la surface de la parcelle.

\subsubsection{Le matériel tensio-capacitif}

Deux rangées de cinq tensiomètres à mercure ont été installées, dans le sens de la pente, à $20 \mathrm{~cm}$ du bord de la parcelle. Les tensiomètres sont espacés de $10 \mathrm{~cm}$ et installés aux profondeurs $5,10,15,25$ et $35 \mathrm{~cm}$. L'ordre d'implantation est inversé entre les deux rangées pour tenir compte d'une éventuelle hétérogénéité spatiale du processus d'infiltration (figure 1). La lecture des niveaux de mercure s'effectue toutes les minutes durant les averses, puis toutes les 5 minutes pendant les phases intermédiaires. La précision de lecture est estimée à $2 \mathrm{hPa}$. L'utilisation de micro-tensiomètres s'étant révélée peu concluante, nous avons utilisé des bougies dont la longueur a été ramenée de 60 à $20 \mathrm{~mm}$ [28]. Malgré un temps d'équilibrage légèrement plus long, ce matériel a permis d'obtenir des mesures satisfaisantes.

Pour mesurer l'humidité du sol quatre capteurs capacitifs ont été implantés, à $10 \mathrm{~cm}$ de profondeur, à chaque extrémité des rangées de tensiomètres (figure l). Un montage électronique, commandé par une centrale d'acquisition de données, a permis de les alimenter de manière cyclique toutes les 30 secondes. La moyenne des mesures de tension électrique est calculée pendant les dix dernières secondes [14].

L'étalonnage des capteurs capacitifs a été réalisé en laboratoire sur monolithe non remanié, dans une enceinte thermostatée $\left(23^{\circ} \mathrm{C}\right)$. Lors de l'expérimentation, la température du sol a varié de 20,5 à $23,5^{\circ} \mathrm{C}$; soit une influence de moins de $0,5 \%$ sur la tension délivrée que nous avons négligée.

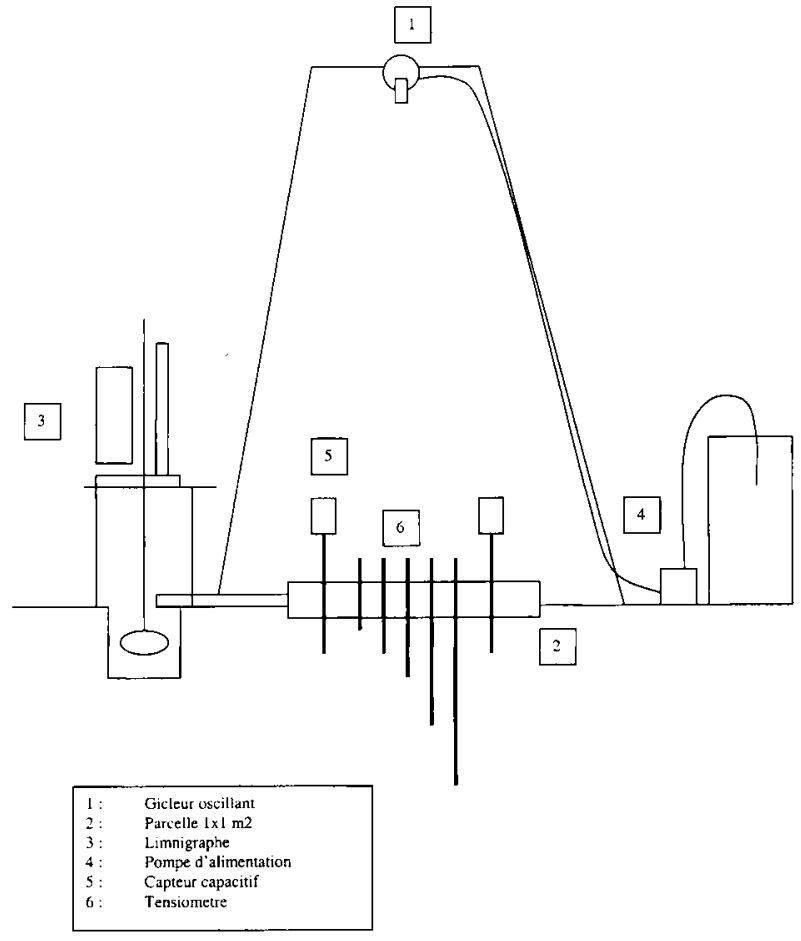

Figure 1. Dispositif expérimental.

\subsubsection{L'infiltromètre à succion contrôlée}

Dans un premier temps, une série de mesures d'infiltration a été réalisée à proximité du site de simulation, sur un sol correspondant à l'état initial. Deux rayons de disque ( $\mathrm{r}: 125$ et $40 \mathrm{~mm}$ ) et deux valeurs du potentiel matriciel $\left(\psi_{0}:-5\right.$ et $\left.-10 \mathrm{hPa}\right)$ ont été utilisés. Trois répétitions ont été effectuées pour chaque couple $\left(r, \psi_{0}\right)$. Le dispositif est déplacé après chaque mesure afin de conserver des conditions initiales équivalentes. Une couche de sable de un centimètre d'épaisseur a été utilisée comme matériau de contact entre le sol et le disque. Initialement, ce protocole devait permettre de calculer les valeurs de la conductivité hydraulique $\left(\mathrm{K}\right.$ en $\left.\mathrm{LT}^{-1}\right)$ à partir de l'analyse du régime permanent $[3,25]$.

Lors de la seconde série de mesures, réalisée une semaine après la simulation de pluies, une croûte superficielle était nettement apparue. Comme Vandevaere et al. [30], nous avons alors restreint l'étude au régime transitoire d'infiltration et choisi de travailler avec un seul disque $(r: 125 \mathrm{~mm})$ et quatre valeurs de potentiel $\left(\psi_{0}\right.$ : $-2,-5,-7,5$ et $-10 \mathrm{hPa}$ ). Trois répétitions ont été effectuées par couple $\left(\mathrm{r}, \psi_{0}\right)$, le dispositif étant, là encore, 
déplacé à chaque fois. Nous avons utilisé la formulation du débit d'infiltration proposée par Warrick [32] :

$$
\mathrm{Q} / \pi \mathrm{r}^{2}=0,5 \cdot \mathrm{S} \cdot \mathrm{t}^{-1 / 2}+\left(0,885 \cdot \mathrm{b}^{1 / 2} \cdot \mathrm{S}^{2}\right) / \mathrm{r} \cdot\left(\Theta_{\mathrm{o}}-\Theta_{n}\right)^{2}
$$

L'intégration par rapport au temps donne [10] :

$$
\mathrm{IC}=\mathrm{S} \cdot \mathrm{t}^{1 / 2}+\left(0,885 \cdot \mathrm{b}^{1 / 2} \cdot \mathrm{S}^{2}\right) \mathrm{t} / \mathrm{r} \cdot\left(\Theta_{0}-\Theta_{n}\right)^{2}
$$

avec : $Q$ débit d'infiltration $\left(\mathrm{L}^{3} \mathrm{~T}^{-1}\right)$

$r$ rayon du disque $(L)$

$\mathrm{S}$ sorptivité capillaire $\left(\mathrm{LT}^{-1 / 2}\right)$

$\mathrm{t}$ temps $(\mathrm{T})$

$\Theta_{0}$ teneur en eau correspondant à $\mathrm{y}_{0}\left(\mathrm{~L}^{3} \mathrm{~L}^{-3}\right)$

$\Theta_{n}$ teneur en eau initiale $\left(\mathrm{L}^{3} \mathrm{~L}^{-3}\right)$

IC infiltration cumulée ( $\mathrm{L}$ )

$\mathrm{b}=0,55[33]$

L'équation quadratique en $S(4)$ possède une racine positive :

$$
\begin{gathered}
\mathrm{S}=\left(\left(1+2,63 \cdot \mathrm{IC} / \mathrm{r} \cdot\left(\Theta_{\mathrm{o}}-\Theta_{n}\right)^{2}\right)^{1 / 2}-1\right) . \\
\left(\mathrm{r} \cdot\left(\Theta_{\mathrm{o}}-\Theta_{n}\right)^{2} \cdot \mathrm{t}^{-1 / 2}\right) / 1,31
\end{gathered}
$$

Les données expérimentales $\mathrm{IC}(\mathrm{t})$ permettent de calculer, d'après (5), la sorptivité capillaire pour les conditions initiales $\left(\Theta_{n}\right)$ et finales $\left(\Theta_{0}\right)$ étudiées. Cette solution semble satisfaisante pour la première heure d'infiltration $[10,15]$. On peut alors accéder au potentiel de flux matriciel selon la relation proposée par White et Sully [33] :

$$
\Phi(\psi)=\mathrm{b} . S^{2} /\left(\Theta_{\mathrm{o}}-\Theta_{n}\right)
$$

En retenant la forme exponentielle de la relation $\mathrm{K}(\psi)=\mathrm{K}_{\mathrm{s}} \mathrm{e}^{\alpha \psi}$ proposée par Gardner [13], on obtient par définition du potentiel de flux matriciel (2):

$$
\Phi(\psi)=\left(\mathrm{K}_{\mathrm{s}} / \alpha\right) \cdot \mathrm{e}^{\alpha \cdot \psi}
$$

L'ajustement de (7) aux valeurs expérimentales $(\Phi, \alpha)$ permet de déterminer les coefficients $\mathrm{K}_{\mathrm{s}}$ et $\alpha$, et donc de connaître la relation entre la conductivité hydraulique et le potentiel matriciel.

\subsubsection{Porosimètre à mercure}

Quatre prélèvements de sol non remanié ont été réalisés avant la simulation (état initial), puis trois autres en fin d'expérimentation dans la parcelle de ruissellement (état final). Les échantillons ont été prélevés en surface grâce à des cylindres de $100 \mathrm{~cm}^{3}$, puis passés à l'étuve $\left(24 \mathrm{~h}\right.$ à $\left.105^{\circ} \mathrm{C}\right)$. Le retrait moyen est de l'ordre de $3 \%$. De chaque cylindre de sol, quatre échantillons $\left(20 \times 10 \times 5 \mathrm{~mm}^{3}\right)$ ont été extraits, deux au sommet (Croûte) et deux au milieu (Massif), afin d'être analysés par porosimétrie au mercure [20]. En application de la loi de Jurin, nous avons obtenu 28 courbes d'intrusion mettant en relation le volume de mercure injecté $\left(\mathrm{V}_{\mathrm{p}}\right)$ avec le diamètre de pore équivalent $\left(\mathrm{D}_{\mathrm{eq}}\right)$ de l'échantillon. Le volume total d'intrusion mesuré $\left(V_{t}\right)$ permet ainsi de calculer les valeurs de la porosité au mercure $\left(\mathrm{n}_{\mathrm{Hg}_{\mathrm{s}}}\right)$ et du diamètre équivalent médian $\left(\mathrm{D}_{\text {med }}\right)[12,19]$. Compte tenu du faible taux d'argile des materiaux utilisés, cette méthode permet de caractériser la porosité d'arrangement des grains et des pores de taille supérieure à $1 \mu \mathrm{m}$. L'effet de retrait a été négligé.

\section{Résultats et discussions}

\subsection{Suivi de l'infiltration sous pluie simulée}

Lors de la première averse à $40 \mathrm{~mm} \cdot \mathrm{h}^{-1}$, le ruissellement apparaît à l'exutoire à $\mathrm{t}=0,22 \mathrm{~h}$ après le début de l'arrosage (figure $2 a$ ). Durant la première heure, la capacité d'infiltration diminue jusqu'à une valeur proche de $25 \mathrm{~mm} . \mathrm{h}^{-1}$. À la reprise de la seconde averse $\left(I=120 \mathrm{~mm} \cdot \mathrm{h}^{-1}\right)$, le ruissellement est presque instantané et le flux d'infiltration s'établit à une valeur proche de la précédente $(F \approx$ $27 \mathrm{~mm} \cdot \mathrm{h}^{-1}$ ). Lors de la seconde pluie, après $21 \mathrm{~h}$ de ressuyage, le ruissellement apparaît à $\mathrm{t}=23,07 \mathrm{~h}$ (figure $2 b$ ). Le flux d'infiltration se stabilise rapidement autour de la valeur $\mathrm{F} \approx 16 \mathrm{~mm} \cdot \mathrm{h}^{-1}$. À la reprise de la seconde averse $\left(120 \mathrm{~mm} \cdot \mathrm{h}^{-1}\right)$, l'infiltration atteint une valeur qui varie très peu jusqu'à la fin de l'expérimentation $\left(\mathrm{F} \approx 14 \mathrm{~mm} \cdot \mathrm{h}^{-1}\right)$.

L'essentiel des modifications dans l'horizon superficiel s'est donc produit pendant la première heure de la première averse. Globalement, le taux de ruissellement passe de $53 \%$ à $70 \%$ entre les deux pluies simulées.

Une heure après le début de la première averse, les tensiomètres et les capteurs placés à l'aval de la parcelle indiquent une stabilisation des valeurs du potentiel matriciel aux cotes $\mathrm{z}=5 \mathrm{~cm}(\psi=$ $-10 \mathrm{hPa}), \mathrm{z}=10 \mathrm{~cm}(\psi=-15 \mathrm{hPa})$ et de la teneur en eau à cette dernière cote $\left(\Theta=0,214 \mathrm{~cm}^{3} \cdot \mathrm{cm}^{-3}\right)$. En revanche, au même instant, les instruments placés à l'amont n'enregistrent aucune stabilisation et les valeurs mesurées correspondent à un profil moins humecté $(\psi=-41 \mathrm{hPa}$ à $5 \mathrm{~cm} ; \psi=-42 \mathrm{hPa}$ 
2a

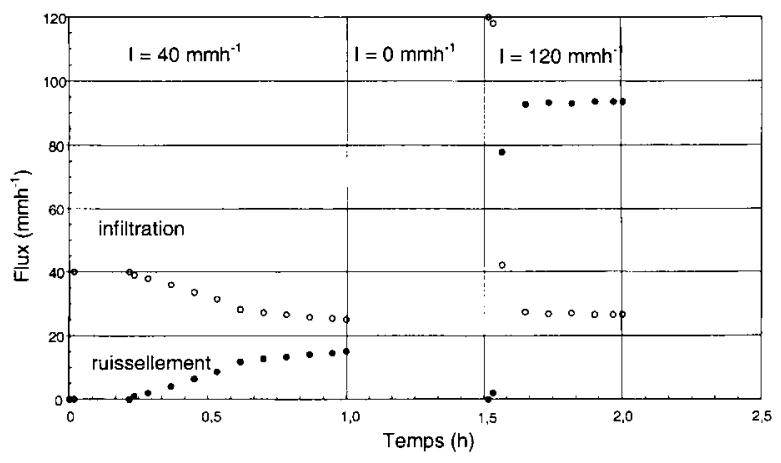

$2 \mathrm{~b}$

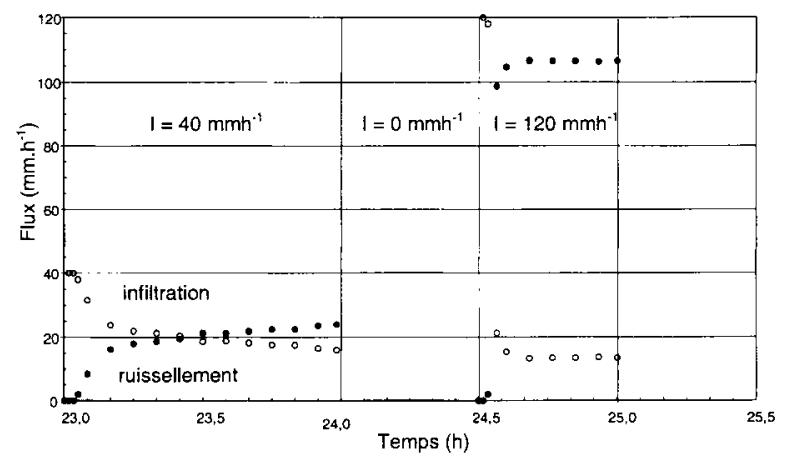

Figure 2. Évolutions des flux de ruissellement et d'infiltration durant la première $(2 \mathrm{a})$ puis la seconde pluie $(2 \mathrm{~b})$.

à $10 \mathrm{~cm} ; \Theta=0,184 \mathrm{~cm}^{3} \cdot \mathrm{cm}^{-3}$ à $\left.10 \mathrm{~cm}\right)$. Cette variabilité, liée à l'organisation progressive du ruissellement de surface, diminue fortement dès la fin de la première averse et devient pratiquement négligeable lors de la seconde. A la fin de la première pluie (figure 3), le comportement hydrodynamique de la parcelle est stabilisé.

À la fin de la seconde pluie, les valeurs moyennes du potentiel matriciel et de teneur en eau, à $10 \mathrm{~cm}$ de profondeur, sont les suivantes : $\psi=$ $-15 \mathrm{hPa}$ et $\Theta=0,220 \mathrm{~cm}^{3} \cdot \mathrm{cm}^{-3}$.

A la suite de Touma [28], nous pouvons considérer que l'extrapolation des profils moyens de charge hydraulique, déterminés aux temps $\mathrm{t}=2,0 \mathrm{~h}$ puis $\mathrm{t}=24,0 \mathrm{~h}$ et $\mathrm{t}=25,0 \mathrm{~h}$, jusqu'à la surface du sol doit

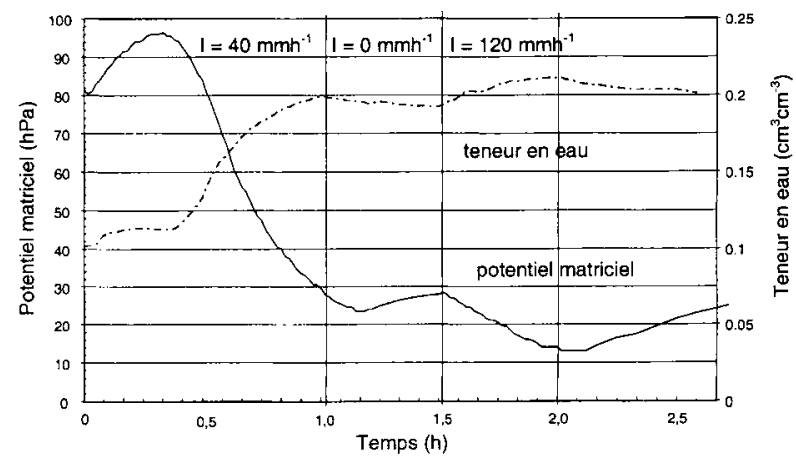

Figure 3. Évolutions de la teneur en eau et du potentiel matriciel à la cote $\mathrm{z}=-10 \mathrm{~cm}$, durant la première pluie.

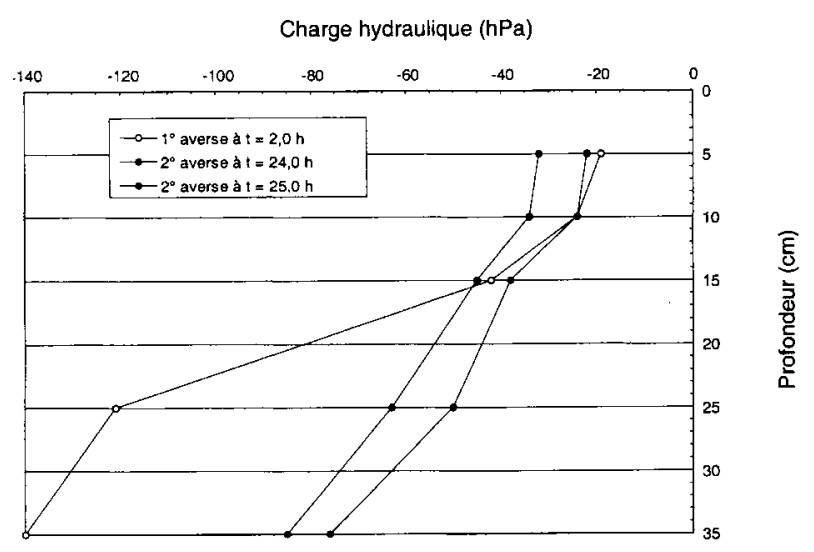

Figure 4. Profils de charge hydraulique obtenus en régime quasi-permanent d'infiltration.

donner une charge nulle à la surface si le sol n'est pas encroûté. En revanche, une charge négative est un indicateur certain de l'existence d'une croûte (figure 4). Dans ce cas, en considérant une croûte établie et un régime d'infiltration quasi-permanent, nous pouvons obtenir une estimation de la conductivité hydraulique de la croûte [16]:

$$
\mathrm{F}=-\mathrm{K}_{\mathrm{c}} \cdot\left(\psi_{\mathrm{mc}}-\mathrm{L}_{\mathrm{c}}-\psi_{\mathrm{cs}}\right) / \mathrm{L}_{\mathrm{c}}
$$

avec : $\mathrm{K}_{\mathrm{c}}$ conductivité hydraulique de la croûte $\left(\mathrm{LT}^{-1}\right)$

$\psi_{\mathrm{mc}}$ potentiel matriciel à la base de la croûte (L) 
Tableau I. Valeurs de la conductivité hydraulique $\left(\mathrm{K}_{\mathrm{c}}\right)$ et de la résistance hydraulique $\left(\mathrm{R}_{\mathrm{c}}\right)$ de la croûte, calculées [equ. 8] à partir de trois valeurs du flux d'infiltration $(\mathrm{F})$ et de potentiel matriciel $\left(\psi_{\mathrm{mc}}\right)$ déterminées expérimentalement.

\begin{tabular}{lccccc}
\hline & $\mathrm{F}\left(\mathrm{mm} \cdot \mathrm{h}^{-1}\right)$ & $\mathrm{dH} / \mathrm{dz}$ à $\mathrm{z}=10 \mathrm{~cm}$ & $\Psi_{\mathrm{mc}}(\mathrm{hPa})$ & $\mathrm{K}_{\mathrm{c}}\left(\mathrm{mm} \mathrm{h}^{-1}\right)$ & $\mathrm{R}_{\mathrm{c}}(\mathrm{h})$ \\
\hline $1^{\circ}$ averse $\mathrm{t}=2,0 \mathrm{~h}$ & 27 & 2,3 & -15 & 0,36 & 5,5 \\
$2^{\circ}$ averse $\mathrm{t}=24,0 \mathrm{~h}$ & 16 & 1,3 & -30 & 0,11 & 18,2 \\
$2^{\circ}$ averse $\mathrm{t}=25,0 \mathrm{~h}$ & 14 & 1,6 & -20 & 0,14 & 14,3 \\
\hline
\end{tabular}

$\psi_{\text {cs }}$ potentiel matriciel à la surface de la croûte (L)

$\mathrm{L}_{\mathrm{c}}$ épaisseur moyenne de la croute $(\mathrm{L})$

La faible épaisseur de la lame d'eau en surface permet de faire l'approximation $\psi_{\mathrm{cs}}=0$. Après observation des échantillons non remaniés prélevés en fin d'expérimentation, nous avons estimé que l'épaisseur de la croûte était proche de $2 \mathrm{~mm}$. Le dépouillement graphique des profils de charge (figure 4) indique que les valeurs superficielles de charge sont nettement négatives. Le tableau I fournit les valeurs de conductivité hydraulique $\left(K_{c}\right)$ et de résistance hydraulique $\left(\mathrm{R}_{\mathrm{c}}=\mathrm{L}_{\mathrm{c}} / \mathrm{K}_{\mathrm{c}}\right)$ pour les trois estimations de $\psi_{\mathrm{mc}}:-15 \mathrm{hPa}(\mathrm{t}=2,0 \mathrm{~h}),-30 \mathrm{hPa}$ $(\mathrm{t}=24,0 \mathrm{~h})$ et $-20 \mathrm{hPa}(\mathrm{t}=25,0 \mathrm{~h})$. La croûte établie est donc caractérisée par une résistance finale de $14,3 \mathrm{~h}$ et une conductivité finale de $0,14 \mathrm{~mm} \cdot \mathrm{h}^{-1}$. Considérant une précision de $\pm 5 \mathrm{hPa}$ pour $\psi_{\mathrm{mc}}$, de $\pm 1 \mathrm{~mm}$ pour $\mathrm{L}_{\mathrm{c}}$ et de $\pm 2 \mathrm{~mm} \cdot \mathrm{h}^{-1}$ pour $\mathrm{F}$, on obtient une erreur globale de $\pm 4,3 \mathrm{~h}$ sur $R_{c}$ et de $\pm 0,12 \mathrm{~mm} \cdot \mathrm{h}^{-1}$ sur $\mathrm{K}_{\mathrm{c}}$.

La croûte superficielle crée une barrière hydraulique qui gouverne le régime d'infiltration et impose une condition non saturante dans l'horizon sousjacent. Pour des sols ferrugineux de texture voisine, Stroosnijder et Hoogmoed [26] indiquent une valeur de flux d'infiltration stabilisée de $9 \mathrm{~mm} \cdot \mathrm{h}^{-1}$ et Valentin [29] fournit une valeur voisine de $17 \mathrm{~mm} \cdot \mathrm{h}^{-1}$. De manière expérimentale, Vandevaere et al. [30] estiment la résistance hydraulique d'une croûte structurale à une valeur de 9 h. Touma [28] trouve une valeur de $10 \mathrm{~h}$ par analyse numérique à partir d'un sol aux caractéristiques hydrodynamiques comparables à celles de notre étude.

\subsection{Caractérisations hydrodynamiques initiale et finale}

La méthode initialement choisie pour caractériser l'état initial (régime permanent) a conduit à des résultats non exploitables, le calcul de la conductivité hydraulique pour $\psi_{0}=-5 \mathrm{hPa}$ donnant une valeur négative. Nous avons donc effectué une analyse du régime transitoire, malgré le nombre limité de valeurs de potentiel testées.

Les lames d'eau infiltrées cumulées permettent de calculer le second membre de l'équation (5). Pour chaque répétition, le potentiel de flux matriciel $(\Phi)$ est alors donné par l'équation (6). L'ajustement aux valeurs moyennes de $\Phi$, selon l'équation (7), donne accès à la conductivité hydraulique $(\mathrm{K})$.

Le tableau II fournit les valeurs de $\Theta_{o}, \Theta_{n}, \mathrm{~S}, \Phi$, $\mathrm{K}_{\mathrm{s}}$ et $\alpha$ établies avant et après la simulation de pluie. La précision sur les valeurs de $S$, obtenues par régression sur les dix premiers points de mesure, est satisfaisante. En revanche la dispersion entre répétitions est nettement plus forte, comme en témoignent les écart-types associés.

L'apparition de la croûte superficielle se traduit donc nettement par une chute d'un facteur 10 environ de la conductivité hydraulique à saturation $\left(\mathrm{K}_{\mathrm{s}}\right)$, qui passe d'une valeur de $107 \mathrm{~mm} . \mathrm{h}^{-1}$ à $11 \mathrm{~mm} . \mathrm{h}^{-1}$ (figure 5). Il est intéressant de noter que cette dernière valeur est proche du flux d'infiltration final enregistré lors de la seconde pluie simulée $(F \approx$ $\left.14 \mathrm{~mm} \cdot \mathrm{h}^{-1}\right)$. Elle correspond également à la conductivité calculée à partir de la relation $\mathrm{K}(\psi)$ initiale, pour une valeur de $\psi=-16 \mathrm{hPa}$ voisine du potentiel matriciel $\psi_{\mathrm{mc}}$ estimé en fin d'expérimen- 
Tableau II. Caractérisation hydrodynamique des états initiaux et finaux pour différentes valeurs de pression appliquées à la surface $\left(\psi_{\mathrm{o}}\right)_{n}$ : teneur en eau initiale; $\Theta \mathrm{o}:$ teneur en eau correspondant à $\psi_{\mathrm{o}} ; \mathrm{S}:$ sorptivité capillaire ; $\Phi$ : potentiel de flux matriciel ; $\mathrm{K}_{\mathrm{s}}$ et $\alpha$ : paramètres de forme de l'équation de Gardner. Entre parenthèses : écart-type associé.

\begin{tabular}{lcccccc}
\hline & \multicolumn{4}{c}{ Etat initial } & \multicolumn{4}{c}{ Etat final } \\
\hline $\mathrm{y}_{\mathrm{o}}(\mathrm{hPa})$ & -5 & -10 & -2 & -5 & $-7,5$ & -10 \\
$\Theta n\left(\mathrm{~cm}^{3} \cdot \mathrm{cm}^{-3}\right)$ & $0,003(0,001)$ & $0,003(0,001)$ & $0,004(0,001)$ & $0,004(0,001)$ & $0,004(0,001)$ & $0,004(0,001)$ \\
$\Theta o\left(\mathrm{~cm}^{3} \cdot \mathrm{cm}^{-3}\right)$ & $0,257(0,030)$ & $0,229(0,026)$ & $0,200(0,034)$ & $0,191(0,016)$ & $0,167(0,020)$ & $0,173(0,006)$ \\
$\mathrm{S}\left(\mathrm{mm}_{\mathrm{s}} \mathrm{s}^{1 / 2}\right)$ & $0,697(0,140)$ & $0,461(0,176)$ & $0,226(0,075)$ & $0,213(0,069)$ & $0,194(0,060)$ & $0,123(0,015)$ \\
$\Phi\left(\mathrm{mm}^{2} . \mathrm{s}\right)$ & $1,064(0,311)$ & $0,531(0,408)$ & $0,149(0,081)$ & $0,134(0,078)$ & $0,131(0,066)$ & $0,050(0,018)$ \\
$\mathrm{K}_{\mathrm{s}}\left(\mathrm{mm}^{-1}\right)$ & 106,68 & & 10,64 & & & \\
$\alpha\left(\mathrm{mm}^{-1}\right)$ & 0,0139 & & 0,0132 & & & \\
\hline
\end{tabular}

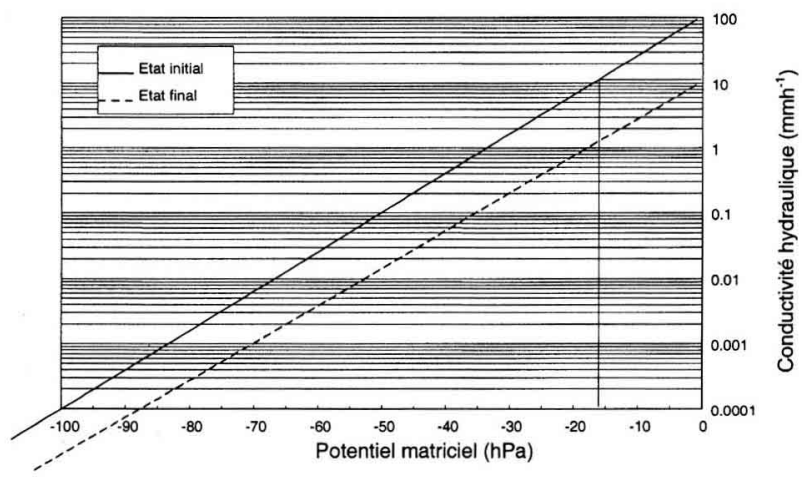

Figure 5. Relations entre la conductivité hydraulique $(\mathrm{K})$ et le potentiel matriciel $(\psi)$ avant et après les deux averses artificielles.

tation $(-15 \mathrm{hPa})$. En revanche, cette valeur de conductivité présente un écart de deux ordres de grandeur avec la conductivité de croûte déterminée expérimentalement $\left(\mathrm{Kc} \approx 0,14 \mathrm{~mm} \cdot \mathrm{h}^{-1}\right)$.

La solution analytique proposée par Warrick [32] ne tenant pas compte de l'effet de la gravité, il nous a paru important de vérifier que celle-ci pouvait être négligée durant la période transitoire utilisée pour le dépouillement des données d'infiltration $(0,25 \mathrm{~h}$ maximum). Une estimation du temps à partir duquel la gravité domine le processus d'infiltration a été donnée par Philip [22] :

$$
\mathrm{t}_{\text {grav }}=(\mathrm{S} / \mathrm{K})^{2}
$$

En fonction du potentiel matriciel appliqué, les valeurs de $\mathrm{t}_{\text {grav }}$ varient de 0,6 à $1,1 \mathrm{~h}$ pour l'état ini- tial. Ces valeurs sont assez faibles et peuvent expliquer la dispersion observée autour des valeurs de $S$ et $\Phi$ (tableau $I)$ ). A l'état final, les valeurs de $t_{\text {orav }}$ varient de 2,7 à $8,7 \mathrm{~h}$. La solution de Warrick [32] semble donc s'appliquer convenablement. Cependant, la durée de la phase transitoire, même réduite, semble disproportionnée par rapport à l'épaisseur de la croûte étudiée $\left(\mathrm{z}_{\mathrm{c}}=2 \mathrm{~mm}\right)$. En fait, la sorptivité puis la conductivité hydraulique ainsi calculées rendent compte du comportement d'un milieu complexe composé de la croûte superficielle et du massif de sol sous-jacent. On comprend dès lors que cette conductivité à saturation soit très supérieure à l'estimation de la conductivité de croûte $\left(\mathrm{K}_{\mathrm{c}}\right)$, mais cohérente avec le débit d'infiltration (F) obtenus en fin de simulation de pluie.

\subsection{Caractérisations de l'espace poral initial et final}

Les valeurs moyennes du volume d'intrusion $\left(\mathrm{V}_{\mathrm{t}}\right)$, du diamètre équivalent médian $\left(\mathrm{D}_{\text {med }}\right)$ et de la porosité au mercure $\left(\mathrm{n}_{\mathrm{Hg}}\right)$ issues de l'analyse par porosimétrie sont reportées dans le tableau III. Concernant l'état initial, aucune différence significative n'est décelable entre les échantillons de Croûte et de Massif. En revanche, l'état final est caractérisé par une une diminution significative du diamètre médian $(20,2 \mu \mathrm{m})$ de la Croûte et, dans une moindre mesure, de sa porosité totale $\left(0,37 \mathrm{~cm}^{3} \mathrm{~cm}^{-3}\right)$. 
Tableau III. Caractérisation de l'espace poral initial et final pour différents échantillons pris en surface (Croûte) ou plus en profondeur (Massif) et soumis à une analyse par porosimétrie au mercure. $\mathrm{V}_{1}$ : volume d'intrusion total ; $\mathrm{D}_{\text {med }}:$ diamètre équivalent médian ; $\mathrm{n}_{\mathrm{Hg}}$ : porosité. Entre parenthèses : écart-type associé.

\begin{tabular}{lcccc}
\hline & & $\mathrm{V}_{\mathrm{t}}\left(\mathrm{cm}^{3} \cdot \mathrm{g}^{-1}\right)$ & $\mathrm{D}_{\text {med }}(\mu \mathrm{m})$ & $\mathrm{n}_{\mathrm{Hg}}\left(\mathrm{cm}^{3} \cdot \mathrm{cm}^{-3}\right)$ \\
\hline État initial & croûte & $0,240(0,017)$ & $28,0(7,4)$ & $0,39(0,02)$ \\
Huit répétitions & massif & $0,248(0,022)$ & $29,8(8,4)$ & $0,40(0,02)$ \\
État final & croûte & $0,227(0,008)$ & $20,2(4,7)$ & $0,37(0,01)$ \\
Six répétitions & massif & $0,252(0,018)$ & $32,8(7,1)$ & $0,40(0,02)$ \\
\hline
\end{tabular}

La représentation de la relation entre la pente de la courbe d'intrusion $\left(\mathrm{dV}_{\mathrm{p}} / \mathrm{d}\left(\log \mathrm{D}_{\mathrm{eq}}\right)\right)$ et le diamètre équivalent $\left(\mathrm{D}_{\mathrm{eq}}\right)$ permet de visualiser l'étalement et le mode de la fonction de répartition des pentes d'intrusion $[11,20]$. Les valeurs modales, ou pics d'intrusion, sont très proches dans le cas initial. L'état final est caractérisé par un décalage entre les deux pics ; cependant, cette différence reste mineure (figure 6). Compte tenu du faible nombre de répétitions, il est difficile d'interpréter l'accroissement relatif du volume correspondant à des pores plus petits (1 à $10 \mu \mathrm{m}$ ) dans les échantillons de croûte. En l'absence d'analyse micromorphologique, l'hypothèse d'un tri granulométrique entre sable fin et limon grossier ne peut être validée. Il faut également noter que cette caractérisation est approximative puisque les échantillons présentent une épaisseur supérieure à celle de la croûte en place.

\section{Conclusion}

Cette expérimentation a permis de démontrer in situ que les processus de réorganisation qui affectent la surface d'un sol ferrugineux sableux entrầnent la formation rapide d'une barrière hydraulique puissante. Au delà de l'incertitude liée aux données expérimentales, la croûte superficielle possède une conductivité hydraulique à saturation inférieure de trois ordres de grandeur à celle du sol en place. À la base de cette croûte, les hypothèses de saturation hydrique et de gradient de charge unitaire ne sont pas vérifiées, ce qui exclut la possibilité d'assimiler

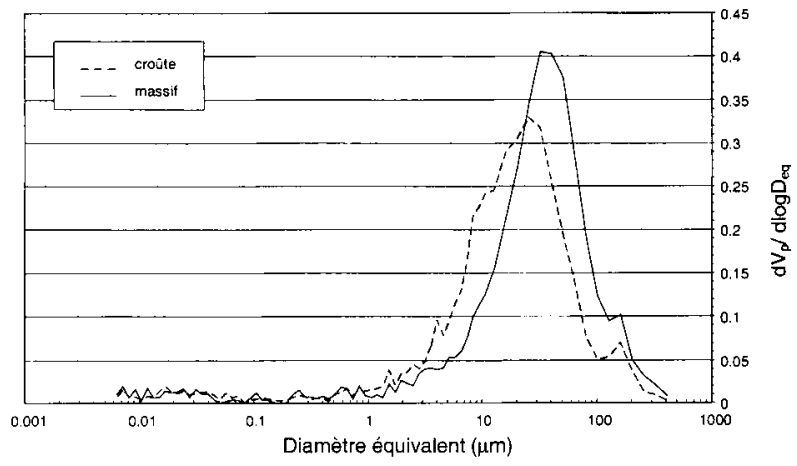

Figure 6. Répartition des pentes de la courbe d'intrusion $\left(\mathrm{dV}_{\mathrm{p}} / \mathrm{d} \log \mathrm{D}_{\mathrm{eq}}\right)$ en fonction du diamètre équivalent $\left(\mathrm{D}_{\mathrm{eq}}\right)$. État

le flux d'infiltration stabilisé, mesuré sous pluie simulée, à une quelconque conductivité hydraulique à saturation, aussi bien du sol que de la croûte qui le surmonte.

Cependant, il est intéressant d'observer que cette valeur est proche de la conductivité hydraulique à saturation calculée à partir des essais d'infiltrométrie à succion contrôlée réalisés en fin d'expérience (État final). Cette valeur de Ks ne caractérise donc pas la croûte proprement dite mais un ensemble constitué de la croûte et du massif de sol sousjacent.

Elle correspond également à la conductivité calculée dans les conditions initiales, pour une valeur du potentiel matriciel comparable à la valeur expérimentale extrapolée à la base de la croûte $\left(\mathrm{h}_{\mathrm{mc}}\right)$. Si cette similitude se confirmait lors de prochains 
essais, il serait alors possible de déduire la valeur de la résistance hydraulique de la croûte à partir de deux séries de mesures par infiltrométrie à succion contrôlée. L'obtention relativement simple de ce paramètre permettrait ainsi d'étendre l'utilisation des modèles d'infiltration à de nombreux sols encroûtés.

Enfin, les résultats obtenus grâce à l'analyse par porosimétrie au mercure semblent indiquer une modification de l'espace poral au sein de la croûte qui s'est formée. Cependant, la texture très homogène du matériau et la faible épaisseur de la croûte rendent l'analyse délicate. Une étude micromorphologique devrait être associée aux analyses de laboratoire afin de mettre en évidence un éventuel tri granulométrique.

Remerciements : Les auteurs tiennent à remercier l'équipe scientifique de l'Institut sénégalais de recherches agricoles, dirigée par le Dr M. Sene, dont la compétence a permis la réalisation et le succès de cette expérimentation.

\section{Références}

[1] Albergel J., Perez P., Vaksmann M., Amélioration des modèles de bilan hydrique sur parcelle par la prise en considération des états de surface, in : Sivakumar M.V.K., Wallace J.S., Renard C. (éds.), Soil water balance in the soudano-sahelian zone, Proc. Niamey workshop, February 1991, IASH Publ. no. 199 (1991) 483-496.

[2] Albergel J., Casenave A., Ribstein P., Valentin C., Aridité climatique, aridité édaphique : études des conditions de l'infiltrabilité en Afrique tropicale sèche, in : Le Floch E., Grouzis M., Cornet A., Bille J.C. (éds.), L'aridité une contrainte au développement, coll. Didactiques, Orstom, Paris, 1992, pp. : 123-131.

[3] Ankeny M.D., Ahmed M., Kaspar T.C., Horton R., Simple field method for determining hydraulic conductivity, Soil Sci. Soc. Am. J. 55 (1991) 467-470.

[4] Bernard A., Un nouveau simulateur de pluie, gouttes et splash, vol. $3 \mathrm{n}^{\circ}$ 2, Orstom Abidjan, 1987, 12-16.

[5] Bertrand R., Morphopédologie et orientations culturales des régions soudaniennes du Sine-Saloum, Sénégal, Agron. Trop. 27 (1972) 1115-1190.
[6] Bresler E., Kemper W.D., Soil water evaporation as affected by wetting methods and crust formation. Soil Sci. Soc. Am. Proc. 34 (1970) 3-8.

[7] Casenave A., Le mini-simulateur de pluie : conditions d'utilisation et principes de l'interprétation des mesures, Cah. Orstom, sér. Hydrol. vol. XIX (1982) 207-227.

[8] Casenave A., Valentin C., Les états de surface de la zone sahélienne. Influence sur l'infiltration, coll. Didactiques, Orstom, Paris, 1989, 226 p.

[9] Casenave A., Valentin C., Influence des états de surface sur l'infiltration en zone sahélienne, in : Sivakumar M.V.K., Wallace J.S., Renard C. (éd.), Soil water balance in the soudano-sahelian zone, Proc. Niamey workshop, February 1991, IASH Publ. no. 199 (1991) 99-108

[10] Cook F.J., Broeren A., Six methods for determining sorptivity and hydraulic conductivity with disc permeameters, Soil Sci. 157 (1994) 2-11.

[11] Fies J.C., Zimmer D., Étude expérimentale de modifications de l'assemblage textural d'un matériau sablo-argileux sous l'effet des pressions, Bulletin du GFHN n ${ }^{\circ}$ 12, Inra Avignon, 1982, 40-54.

[12] Fies J.C., Analyse de la répartition des pores dans les assemblages argile-squelettes : comparaison entre un modèle d'espace poral textural et les données fournies par la porosimétrie au mercure, Agronomie 4 (1984) 891-899.

[13] Gardner W.R., Some steady state solutions of the unsaturated moisture flow equation with applications to evaporation from a water table, Soil Sci. 85 (1958) 228-232.

[14] Gaudu J.C., Mathieu J.M., Fumanal J.C., Bruckler L., Mesure de l'humidité des sols par une méthode capacitive : analyse des facteurs influençant la mesure, Agronomie 13 (1993) 57-73.

[15] Haverkamp R., Ross P.J., Smettem K.R.J., Parlange J.Y., Three dimensional analysis of infiltration from the disc infiltrometer. 2. Physically based infiltration equation, Water Resour. Res. 30 (1994) 2931-2935.

[16] Hillel D., Gardner W.R., Transient infiltration into crusted-topped profiles, Soil Sci. 109 (1970) 69-76.

[17] Hoogmoed W.B., Stroosnijder L., Crust formation on sandy soils in the Sahel. Part I : rainfall and infiltration, Soil Tillage Res. 4 (1984) 5-23.

[18] Mc Intyre D.S., Permeability measurements of soil crusts formed by raindrop impact, Soil Sci. 85 (1958) 185-189. 
[19] Pellerin F.M., La porosimétrie au mercure appliquée à l'étude géotechnique des sols et des roches, Bull. Liais. Lab. des Ponts et Chaussées 106 (1980) 105-116.

[20] Perez P., Genèse du ruissellement sur les sols cultivés du sud Saloum (Sénégal). Du diagnostic à l'aménagement de parcelle, thèse, Ensa Montpellier, France, 1994, $250 \mathrm{p}$.

[21] Perroux K.M., White I., Designs for disk permeameters, Soil Sci. Soc. Am. J. 52 (1988) 1205-1214.

[22] Philip J.R., Theory of infiltration, Adv. Hydrosci. 5 (1969) 215-296.

[23] Philip J.R., The quasilinear analysis, the scattering analog and other aspects of infiltration and seepage, in : Fok Y. S. (éd.), Infiltration development and application. Water Resour. Res. Center, Honolulu, Hawai, 1987, pp. 1-27.

[24] Rawitz E., Hoogmoed W.B., Morin Y., Development of criteria and methods for improving the efficiency of soil management and tillage operations, with special reference to arid and semi-arid regions, Agric. Univ., Wageningen, and Hebrew Univ., Rehovot, 1981.

[25] Smettem K.R.J., Clothier B.J., Measuring unsaturated sorptivity and hydraulic conductivity using multiple disc permeameter, J. Soil Sci. 40 (1989) 563-568.

[26] Stroosnijder L., Hoogmoed W.B., Crust formation on sandy soils in the Sahel. Part II: Tillage and its effect on the water balance, Soil Tillage Res. 4 (1984) 321-337.

[27] Touma J., Albergel J., Determining soil hydrologic properties from rain simulator or double ring infiltrometer experiments. A comparison, J. Hydrol. 135 (1992) 73-86.

[28] Touma J., Simulation numérique de l'infiltration dans les sols encroûtés : croûte établie et en formation, Hydrol. Continent. 7 (1992) 143-156.

[29] Valentin C., Surface crusting in two alluvial soils of northern Niger, Geoderma 48 (1991) 201-222.

[30] Vandervaere J.P., Jaramillo R.A., Peugeot C., Vauclin M., Caractérisation hydrodynamique in situ de sols encroûtés, in : Journées Hydrologiques de L'orstom, Actes du séminaire de Montpellier, 13 et 14 sept. 1994, Orstom, Montpellier, 1994, pp. 603-613.

[31] Vauclin M., Chopart J.L., L'infiltrométrie multidisques pour la détermination in situ des caractéristiques hydrodynamiques de la surface d'un sol gravillonnaire de Côte-d'Ivoire, Agron. Trop. 46-4 (1992) 259-271.

[32] Warrick A.W., Models for disc infiltrometers, Water Resour. Res. 28 (1992) 1319-1327.

[33] White I., Sully M.J., Macroscopic and microscopic capillary length and time scales from field infiltration, Water Resour. Res. 23 (1987) 1514-1522. 